\title{
Viscous sintering of unimodal and bimodal cylindrical packings with shrinking pores
}

\author{
DARREN CROWDY \\ Department of Mathematics, Imperial College of Science, Technology and Medicine, \\ 180 Queen's Gate, London, UK \\ email: d.crowdy@ic.ac.uk
}

(Received 30 July 2001; revised 18 September 2002)

\begin{abstract}
Calculations, based on exact solutions, of the viscous sintering of simple packings with unimodal and bimodal distributions of particle sizes with shrinking pores are performed. The case of square unit cells is considered in detail. It is found that, for the most part, pore shrinkage times have very weak dependence on the precise details of the pore shape and that accurate estimates of total pore shrinkage times can be obtained based only on knowledge of the initial relative density (green density) and unit cell size. An exception is found when the particle packing is loose, the enclosed pore having a large perimeter-to-area ratio. By studying two different square packings of equal-sized particles it is shown that the looser packing can exhibit dilation, rather than densification, in the early stage of sintering.
\end{abstract}

\section{Introduction}

Sintering describes a process by which a granular compact of particles (e.g. metal or glass) is raised to a sufficiently large temperature that the individual particles become mobile and release surface energy in such a way as to produce inter-particulate bonds. At the start of a sinter process, any two particles which are initially touching develop a thin 'neck' which, as time evolves, grows in size to form a more developed bond. In compacts in which the packing is such that particles have more than one touching neighbour, as the necks grow in size, the sinter body densifies and any enclosed pores between particles tend to close up. The macroscopic material properties of the compact at the end of the sinter process depend heavily on the degree of densification. In industrial application, it is crucial to be able to obtain accurate and reliable estimates of the time taken for pores to close (or reduce to a sufficiently small size) within any given initial sinter body in order that industrial sinter times are optimized without compromising the macroscopic properties of the final densified sinter body.

To model the sintering process analytically so that quantitative predictions can be made, it is usual to divide the process into three distinct stages. These are referred to as the initial stage, intermediate stage and final stage and models have been devised to study each separate stage of the process. In all such models, the state of the sinter kinetics is typically described by isolating a typical unit cell in the matrix and making some geometrical simplification. With this simplification, it then becomes possible to define the notion of the relative density of the fluid/solid phase in this unit. The degree of sintering is then 
tracked by using an energy balance argument to give this relative density as a function of time.

In the sintering of amorphous materials under the effects of surface tension the initial stage is characterized by rapid interparticle neck growth while in the intermediate stage these sharp neck regions have been smoothed out leaving a network of inter-connected pores which, by the final stage, has densified to such a degree that the compact is now well modelled by a uniform region of fluid containing small isolated pores. An important initial stage model is the two sphere model due to Frenkel [9]. Frenkel made some major simplifications regarding the flow field to deduce an equation governing the neck-growth rate between two initially-touching spheres as they coalesce. This is done using an energy principle based on equating the viscous flow dissipation with the released surface energy. Scherer [26] introduced an intermediate stage model, referred to as the 'cylinder model', in which the fluid is modelled by an idealized cubic array of intersecting cylinders in which densification is described by the cylinders getting shorter and thicker. With these geometrical simplications, the dynamical evolution of the cylinders is provided by an energy balance argument similar to that used by Frenkel. To model the final stage, Mackenzie \& Shuttleworth [16] considered an idealized structure consisting of spherical pores of the same size in a fluid/solid matrix. It is assumed that a concentric shell consisting of a solid spherical matrix containing an enclosed spherical pore maintains its form under sintering. With these geometrical simplications, the sinter dynamics is again provided by an energy balance principle.

The densification of a sinter body is normally tracked using a quantity known as relative density [18] [1]. Roughly speaking, the relative density is the amount of fluid (as opposed to pore) contained in a typical unit cell. In a fully densified sinter body a unit cell contains only fluid and no pores. Both experimental data, and the predictions of the 3D theoretical models just described typically give rise to a sigmoidal, or S-shaped, relative density-versus-time curve for the sintering of amorphous materials. One of the remarkable facts about the $3 \mathrm{D}$ models is that while different geometrical assumptions are made in describing the separate stages of sintering, the various models are in good agreement over a large range of relative densities and appear to give good estimates of sinter rates even outside their respective ranges of validity. The conclusion is that the viscous sintering of amorphous materials is not particularly sensitive to structural features such as the geometry of the pore shape. One of the objectives of this paper is to use a class of exact solutions describing simple unit cells with shrinking pores to examine whether this phenomenon also pertains to a mathematical model of planar sintering based on the assumption of slow viscous flow driven by surface tension.

In the case of planar sintering the analogue of Frenkel's two-sphere unit problem is the coalescence of two equal cylindrical particles. The two-dimensional viscous sintering problem has received a revival of theoretical interest following the work of Hopper [11], who analysed this unit problem and showed that it admits exact solutions in the sense that the full free boundary problem can be reduced to the integration of a set of coupled nonlinear ordinary differential equations. In a natural extension of Hopper's result, Richardson [19] extended the class of exact solutions to the case of two unequal cylinders coalescing. This provides a unit problem modelling the early-stage neck growth at the point of contact of two different sized particles in a bimodal distribution of particles. 
Numerical simulations of multiply-connected planar domains by Van de Vorst [28] suggest that Hopper's unit problem provides an excellent description for the early-stage neck growth in a doubly-connected packing of equal-sized cylindrical particles, the implication being that the exact solution will, in general, provide a good model of neck growth rates between equal-sized particles in an arbitrary compact. The main advantage of Hopper's solution is its mathematical simplicity and the fact that both the boundary evolution and associated flow field are both consistently resolved (i.e. without any a priori geometrical assumptions). One drawback of Hopper's unit problem is that the fluid configuration is simply-connected and thus contains no shrinking pores between the particles so that while the initial stage neck-growth process is captured by the model, it is not possible to infer estimates regarding overall shrinkage rates of inter-particulate pores.

In this paper, we study a class of exact solutions corresponding to generalized unit problems involving shrinking pores which share the virtues of simplicity of Hopper's solution but which capture a more global picture of the sinter process. The problems considered are explicit exact solutions to a class of simple sinter problems involving both unimodal and bimodal particles size distributions surrounding an enclosed pore. These generalized unit problems are therefore capable of describing both the early stage neck growth and the later stage pore shrinkage. The exact solutions are based on a theory devised by Crowdy \& Tanveer [3] in an earlier paper. Even the more complicated bimodal solutions calculated in $\S 4$ of this paper require little more than the numerical integration of three coupled nonlinear ordinary differential equations. Given this simplicity, it is easy to make a large number of calculations in a short time and build a broad picture of the possible behaviour of the system. A principal objective of this paper is to establish how the geometry of the pore shape affects pore disappearance times.

It is important to clarify an assumption which underlies the present paper. By studying exact solutions, various properties of the sintering of isolated unit cells are established. It is a premise of this paper that the behavioural properties of the pores in the isolated unit cell may well be qualitatively similar to those of a pore in a similar unit cell immersed in an extended doubly-infinite lattice. Of course, the extent to which this is true will only be established once the sintering problem for the full lattice is solved. It seems appropriate, however, to document the properties of the isolated unit cell in order to facilitate future comparisons with a cell situated in the full lattice.

We now summarize the class of solutions to be considered and indicate how they generalize previous work on simply-connected fluid regions. For two equal cylinders, Hopper [11] showed the fluid domain under the sinter dynamics can be described by means of a conformal map from a unit $\zeta$-disc of the form

$$
z(\zeta, t)=\frac{A(t)}{\zeta-a(t)}+\frac{A(t)}{\zeta+a(t)}=\frac{2 A(t) \zeta}{\zeta^{2}-a^{2}(t)},
$$

where $a(t)$ and $A(t)$ are two parameters which evolve according to a coupled system of two nonlinear differential equations. Richardson [19] extended this to the case of two unequal cylinders for which the relevant conformal map has the rational function form

$$
z(\zeta, t)=\frac{A(t) \zeta}{\zeta-a(t)}+\frac{B(t) \zeta}{\zeta+b(t)},
$$


where the time-evolving parameters are now $a(t), b(t), A(t)$ and $B(t)$. Later, Richardson [21] generalized [11] in a different direction by considering exact solutions for arbitrary simply-connected unimodal particle distributions consisting of more than two particles. In this paper, two classes of conformal maps generalizing (1.1) and (1.2) are studied in detail. The maps are now from a parametric annulus $\rho(t)<|\zeta|<1$. The map

$$
z(\zeta, t)=A(t) \zeta \frac{P_{N}\left(\zeta \rho^{2 / N} a^{-1}, \rho\right)}{P_{N}\left(\zeta a^{-1}, \rho\right)}, \quad N \geqslant 3,
$$

where $a(t)$ and $A(t)$ are real parameters generalizes (1.1) to the case of a unimodal distribution of $N$ equal cylinders in an annular array. The analogous generalization of (1.2) is given by

$$
z(\zeta, t)=A(t) \zeta \frac{P_{N}\left(\zeta \rho^{2 / N} a^{-1}, \rho\right)}{P_{N}\left(\zeta a^{-1}, \rho\right)}+B(t) \zeta \frac{Q_{N}\left(\zeta \rho^{2 / N} b^{-1}, \rho\right)}{Q_{N}\left(\zeta b^{-1}, \rho\right)},
$$

where $a(t), A(t), b(t)$ and $B(t)$ are real parameters and $N \geqslant 3$ is an integer. (1.4) corresponds to a bimodal distribution of two different sized cylinders in an annular configuration. $P_{N}(\zeta, \rho)$ and $Q_{N}(\zeta, \rho)$ are special functions to be defined later.

Independently, Richardson [22] has used a different formulation involving elliptic function conformal mappings from a rectangular parametric region to find exact solutions for the sintering of a doubly-connected fluid region. Richardson also points out that a particular illustrative example presented in the original theory of Crowdy \& Tanveer [3] is in error as the chosen initial conditions are not consistent with the underlying assumptions of the theory [3]. The authors of that paper agree with him on this [4]; nonetheless, the general theory of Crowdy \& Tanveer [3], and the associated evolution equations, remain valid with appropriate choices of the initial conditions and can be used to determine the evolution of a variety of doubly-connected domains of physical interest, as shall be seen in the examples calculated in this paper. Richardson's alternative formulation [22] can also be used to calculate the examples considered herein.

\section{Doubly-connected exact solutions}

Crowdy \& Tanveer [3] have presented a theory of exact solutions for the problem of the viscous sintering of doubly-connected, annular blobs of viscous fluid. The fluid is modelled as a doubly-connected region $D$ of very viscous incompressible fluid in which a streamfunction $\psi(x, y)$ is governed by the biharmonic equation

$$
\nabla^{4} \psi=0, \quad(x, y) \in D
$$

where the velocity field is given by

$$
\mathbf{u}=(u, v)=\left(\psi_{y},-\psi_{x}\right)
$$

On the boundary $\partial D$ of $D$, the tangential stress must vanish and the normal stress must be balanced by the uniform surface tension effect. In terms of the usual Newtonian stress 
tensor, this condition can be written

$$
-p n_{i}+2 \mu e_{i j}=T \kappa n_{i}
$$

where $p$ is the fluid pressure, $\mu$ is the viscosity, $T$ is the surface tension parameter, $\kappa$ is the boundary curvature, $n_{i}$ denotes components of the outward normal $\mathbf{n}$ to $\partial D$ and

$$
e_{i j}=\frac{1}{2}\left(\frac{\partial u_{i}}{\partial x_{j}}+\frac{\partial u_{j}}{\partial x_{i}}\right) .
$$

At each instant, the above boundary value problem defines a global velocity field which will not, in general, be in equilibrium. The boundary is time-evolved in a quasi-steady fashion with a normal velocity $V_{n}$ determined by the kinematic condition

$$
V_{n}=\mathbf{u . n} .
$$

The reader is referred to the original theory for doubly-connected domains [3] for a detailed exposition. Here only a review of the principal results needed for the calculations of this paper will be presented.

The importance of the theory in Crowdy \& Tanveer [3] is to show that, under the dynamics of viscous sintering with an assumption that two constants of integration arising in the analysis are equal (denoted $A_{0}(t)$ and $A_{I}(t)$ in Crowdy \& Tanveer [3]), conformal mappings from an annulus $\rho(t)<|\zeta|<1$ to the time-evolving annular blob of fluid that are initially meromorphic in the (fundamental) annulus $\rho<|\zeta|<\rho^{-1}$ and satisfying the relation

$$
z\left(\rho^{2} \zeta, t\right)=z(\zeta, t)
$$

remain meromorphic and continue to satisfy (2.6) under evolution. Such functions are known as loxodromic functions. For more details on loxodromic functions, the reader is referred to Valiron [31], or a useful appendix modelled on [31] which appears in Richardson [20].

These results give rise to exact solutions in the sense of closed form formulae for the conformal mapping functions which depend on a finite set of time-evolving parameters. One representation of a meromorphic function $z(\zeta, t)$ satisfying $(2.6)$ is given by

$$
z(\zeta, t)=R \frac{\prod_{j=1}^{n} P\left(\zeta \eta_{j}^{-1}, \rho\right)}{\prod_{j=1}^{n} P\left(\zeta \zeta_{j}^{-1}, \rho\right)}
$$

where $\left\{\zeta_{j} \mid j=1,2, . ., n\right\}$ are the $n$ poles of the mapping in the annulus $\rho^{2}<|\zeta|<1$ and where $\left\{\eta_{j} \mid j=1,2, . ., n\right\}$ are the zeros of the mapping in this annulus. The function $P(\zeta, \rho)$ is defined as

$$
P(\zeta, \rho)=(1-\zeta) \prod_{k=1}^{\infty}\left(1-\rho^{2 k} \zeta\right)\left(1-\rho^{2 k} \zeta^{-1}\right) .
$$

The poles and zeros satisfy the condition

$$
\prod_{j=1}^{n} \eta_{j}=\prod_{j=1}^{n} \zeta_{j}
$$


and they, along with the parameters $R$ and $\rho$, are time-evolving functions whose evolution must be determined as part of the solution. For compactness of notation, explicit dependence of such parameters on time $t$ has been suppressed. The same non-dimensionalization used in Crowdy \& Tanveer [3] is also adopted here.

The general evolution equations for the parameters are derived in Crowdy \& Tanveer [3] although only those relevant to initial conformal maps possessing a finite distribution of simple pole singularities will be reviewed here. The conformal modulus $\rho(t)$ evolves according to the real equation

$$
\dot{\rho}=-\frac{\rho}{4 \pi i}\left(\oint_{\left|\zeta^{\prime}\right|=1} \frac{d \zeta^{\prime}}{\zeta^{\prime}} \frac{1}{\left|z_{\zeta}\left(\zeta^{\prime}, t\right)\right|}+\oint_{\left|\zeta^{\prime}\right|=\rho} \frac{d \zeta^{\prime}}{\zeta^{\prime}} \frac{1}{\rho\left|z_{\zeta}\left(\zeta^{\prime}, t\right)\right|}\right),
$$

while all simple poles evolve according to

$$
\frac{d \bar{\zeta}_{j}^{-1}}{d t}=-\bar{\zeta}_{j}^{-1} I\left(\bar{\zeta}_{j}^{-1}, t\right)
$$

where $I(\zeta, t)$ is defined as

$$
I(\zeta, t)=I^{+}(\zeta, t)-I^{-}(\zeta, t)+C(t)
$$

with

$$
\begin{aligned}
I^{+}(\zeta, t) & =\frac{1}{4 \pi i} \oint_{\left|\zeta^{\prime}\right|=1} \frac{d \zeta^{\prime}}{\zeta^{\prime}}\left(1-2 \frac{\zeta}{\zeta^{\prime}} \frac{P^{\prime}\left(\frac{\zeta}{\zeta^{\prime}}, \rho\right)}{P\left(\frac{\zeta}{\zeta^{\prime}}, \rho\right)}\right) \frac{1}{\left|z_{\zeta}(\zeta, t)\right|}, \\
I^{-}(\zeta, t) & =\frac{1}{4 \pi i} \oint_{\left|\zeta^{\prime}\right|=\rho} \frac{d \zeta^{\prime}}{\zeta^{\prime}}\left(1-2 \frac{\zeta}{\zeta^{\prime}} \frac{P^{\prime}\left(\frac{\zeta}{\zeta^{\prime}}, \rho\right)}{P\left(\frac{\zeta}{\zeta^{\prime}}, \rho\right)}\right)\left[-\frac{1}{\rho\left|z_{\zeta}(\zeta, t)\right|}-\frac{2 \dot{\rho}}{\rho}\right], \\
C(t) & =-\frac{1}{4 \pi i} \oint_{\left|\zeta^{\prime}\right|=\rho} \frac{d \zeta^{\prime}}{\zeta^{\prime}}\left[-\frac{1}{\rho\left|z_{\zeta}(\zeta, t)\right|}-\frac{2 \dot{\rho}}{\rho}\right]
\end{aligned}
$$

Note that the notation $I(\zeta, t)$ disguises the fact that this function depends on the parameters appearing in the conformal map and this should be borne in mind. $P^{\prime}(\zeta, \rho)$ denotes the derivative with respect to the first argument. Finally, all remaining evolution equations follow from what is referred to in Crowdy \& Tanveer [3] as a theorem of invariants. This theorem establishes that the $n$ quantities

$$
B_{j}=\frac{J^{j}(t)}{\prod_{\substack{p=1 \\ p \neq j}}^{n} P\left(\bar{\zeta}_{p} \bar{\zeta}_{j}^{-1}\right)}, \quad j=1,2, . ., n
$$

are invariants of the motion, where

$$
J^{j}(t) \equiv \oint_{\partial C}\left(\prod_{\substack{p=1 \\ p \neq j}}^{n} P(\zeta \bar{\zeta}-1) \bar{z} z_{\zeta}\right) d \zeta,
$$

where $\partial C$ denotes the boundary of the annulus $\rho<|\zeta|<1$ with the outer circle traversed in an anti-clockwise direction, the inner circle in a clockwise direction. 
In a different approach to the same problem, Richardson [22] also recognized the need, if exact solutions are to be found, for two integration constants arising in the integrated stress conditions (cf. $A_{O}(t)$ and $A_{I}(t)$ in Crowdy \& Tanveer [3]) to assume the same value under evolution. Richardson has further pointed out that a sufficient condition for this to be valid is that the physical domain possess a rotational symmetry. We therefore now restrict attention to initial domains described by loxodromic function conformal maps which possess such a symmetry (see also Crowdy \& Tanveer [4]). Such symmetries are preserved in time and the theory developed in Crowdy \& Tanveer [3], and reviewed above, can be conveniently applied as will now be shown.

We now consider two particular classes of loxodromic conformal maps, namely,

$$
z(\zeta, t)=A(t) \zeta \frac{P_{N}\left(\zeta \rho^{2 / N} a^{-1}, \rho\right)}{P_{N}\left(\zeta a^{-1}, \rho\right)},
$$

and

$$
z(\zeta, t)=A(t) \zeta \frac{P_{N}\left(\zeta \rho^{2 / N} a^{-1}, \rho\right)}{P_{N}\left(\zeta a^{-1}, \rho\right)}+B(t) \zeta \frac{Q_{N}\left(\zeta \rho^{2 / N} b^{-1}, \rho\right)}{Q_{N}\left(\zeta b^{-1}, \rho\right)},
$$

and show that they constitute exact solutions to the sintering problem. $N \geqslant 3$ is an integer. Defining $\omega=e^{\frac{2 \pi i}{N}}$ as an $N$-th root of unity, the special function $P_{N}(\zeta, \rho)$ is related to the function $P(\zeta, t)$ already introduced via the formula

$$
\begin{aligned}
P_{N}(\zeta, \rho) & =\prod_{j=0}^{N-1} P\left(\zeta \omega^{j}, \rho\right) \\
& =\left(1-\zeta^{N}\right) \prod_{k=1}^{\infty}\left(1-\rho^{2 k N} \zeta^{N}\right)\left(1-\rho^{2 k N} \zeta^{-N}\right),
\end{aligned}
$$

while the function $Q_{N}(\zeta, \rho)$ is defined as

$$
\begin{aligned}
Q_{N}(\zeta, \rho) & =\prod_{j=0}^{N-1} P\left(\zeta \tilde{\omega}^{j}, \rho\right) \\
& =\left(1+\zeta^{N}\right) \prod_{k=1}^{\infty}\left(1+\rho^{2 k N} \zeta^{N}\right)\left(1+\rho^{2 k N} \zeta^{-N}\right),
\end{aligned}
$$

where $\tilde{\omega}=e^{\frac{i \pi}{N}}$. Note that both $P_{N}(\zeta, \rho)$ and $Q_{N}(\zeta, \rho)$ are purely functions of $\zeta^{N}$ and so, with the pre-multiplying factor of $\zeta$ common to both (2.16) and (2.17), an $N$-fold rotational symmetry is now a built-in feature of the mappings (2.16) and (2.17) for all choices of the parameters appearing in them.

As mentioned in Crowdy \& Tanveer [3], the function $P(\zeta, \rho)$ satisfies the following functional equations:

$$
P\left(\zeta^{-1}, \rho\right)=P\left(\rho^{2} \zeta, \rho\right)=-\zeta^{-1} P(\zeta, \rho) .
$$

These relations are easily checked. (2.20) implies that $P_{N}(\zeta, \rho)$ satisfies

$$
P_{N}\left(\zeta^{-1}, \rho\right)=P_{N}\left(\rho^{2} \zeta, \rho\right)=-\zeta^{-N} P_{N}(\zeta, \rho)
$$



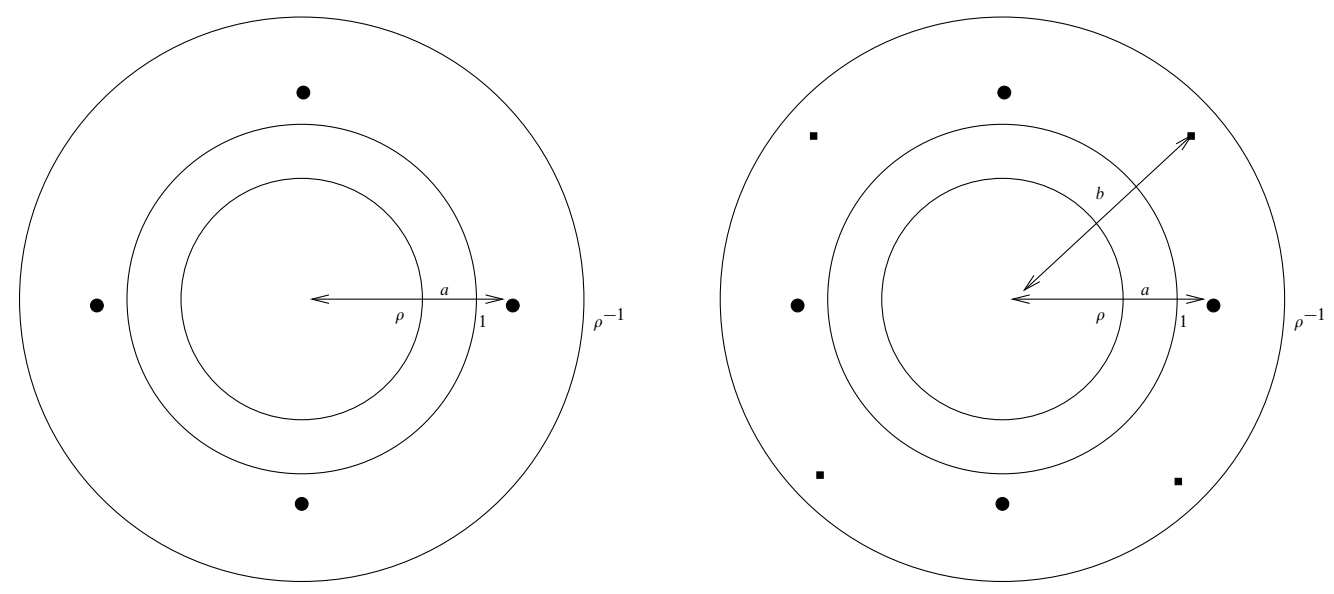

FIGURE 1. Pole distributions in the fundamental annulus for maps (2.16) (left-most diagram) and (2.17) (right-most diagram) with $N=4$. Bold dots and squares indicate the positions of the (simple) poles.

Using (2.21), straightforward algebra reveals that mappings (2.16) and (2.17) satisfy the requirement (2.6) while it is also clear that both functions are meromorphic in the fundamental annulus $\rho<|\zeta|<\rho^{-1}$. These maps are therefore loxodromic functions and are consistent with all the assumptions underlying the theory of Crowdy \& Tanveer [3]. They are therefore exact solutions of the physical problem.

To briefly motivate the choice of formulae (2.16) and (2.17), note that each simple pole of the conformal mapping function in the fundamental annulus $\rho<|\zeta|<\rho^{-1}$ can be understood as corresponding to a particle in the physical domain. In (2.16), $N$ poles are distributed at equispaced angles about the origin all at radial distance $a(t)$. See the left-most schematic in Figure 1, where the poles of the mapping (2.16) in the fundamental annulus for $N=4$ are shown as bold dots. It is therefore natural to expect this map to correspond (for appropriate choices of $a$ and $\rho$ ) to an annular array of equal-sized particles surrounding the origin. In the same way, (2.17) also has $N$ poles at radial distance $a(t)$ (where $1<a(t)<\rho(t)^{-1}$ ) from the origin but with $N$ additional poles at radial distance $b(t)$ (where $1<b(t)<\rho(t)^{-1}$ ) placed between them at equal angular displacements. See the right-most schematic in Figure 1, where the poles of the mapping (2.17) at radial distance $a$ from the origin are drawn as bold dots, but those at distance $b$ are drawn as bold squares. For suitable parameter choices, the map (2.17) can be expected to give a bimodal distribution of particle sizes alternating in an annular array about the origin, the poles shown as dots corresponding to particles of one size, the poles shown as squares to particles of a different size placed between them.

On a practical note, both maps (2.16) and (2.17) as well as the differential equations governing their time evolution involve only the special function $P(\zeta, \rho)$ (and the closely related variants $P_{N}(\zeta, \rho)$ and $Q_{N}(\zeta, \rho)$ ). The maps and evolution equations can therefore easily be programmed into a numerical code (or plotting software) without the need for any pre-programmed special function routines. This can be done by truncating the infinite products (2.18) after a suitably large number of terms (keeping just the first terms 
corresponding to $k=1,2, . ., 10$ is found to provide excellent accuracy in all the calculations that follow).

\section{Unimodal particle packings with pores}

The map (2.16) depends upon just three real parameters $\rho(t), a(t)$ and $A(t)$. From $\S 2$, the three evolution equations required for mappings of the form (2.16) are given by (2.10) the usual equation for $\rho(t)-$ along with the two additional equations

$$
\dot{a}=a I\left(a^{-1}, t\right),
$$

and

$$
\frac{A P_{N}\left(\rho^{2 / N}, \rho\right) z_{\zeta}\left(a^{-1}, t\right)}{N \hat{P}_{N}(1, \rho)}=\text { constant }
$$

where the function $\hat{P}_{N}(\zeta, \rho)$ is defined as

$$
\hat{P}_{N}(\zeta, \rho) \equiv \frac{P_{N}(\zeta, \rho)}{\left(1-\zeta^{N}\right)}=\prod_{k=1}^{\infty}\left(1-\rho^{2 k N} \zeta^{N}\right)\left(1-\rho^{2 k N} \zeta^{-N}\right)
$$

and the constant in (3.2) is simply the value of the left hand side at $t=0$. Equation (3.2) is a consequence of the theorem of invariants of Crowdy \& Tanveer [3] and is equivalent to (2.14). Equations (2.10), (3.1) and (3.2) are solved numerically.

Two of the three example calculations performed by Richardson [22] correspond to the map (2.16) in cases $N=3$ and $N=4$. To facilitate comparison with his results, we choose initial conditions in the same manner. Richardson discusses these initial conditions in terms of a Hele-Shaw analogy but they amount to choices of initial conditions close to the case of a rotationally-symmetric array of $N$ touching unit-radius cylinders in which

$$
z\left(a(0)^{-1}, 0\right)=\frac{1}{\sin \frac{\pi}{N}}-10^{-3},
$$

and such that the midpoint of the two points on the inner and outer boundaries which are closest together is precisely at the point of contact of the configuration of $N$ touching cylinders. The total fluid area is taken to be $N \pi$. These conditions are enough to uniquely specify the initial domain.

The calculations are performed using an explicit Euler method with time-step equal to $10^{-4}$. The computations are stopped when $\rho$ decreases below a threshold of $10^{-4}$ so that the final area of the remaining pore is of the order of $10^{-8}$ (the pore is close to circular just before it disappears). All integrals are performed using a trapezoidal rule with an equally-spaced discretization of 1024 points in the angular coordinate of the unit $\zeta$-circle this gives super-algebraic convergence for smooth periodic functions (which is the case here). The order of the global error in the calculation is expected to be of the same order as the time-step, i.e. $10^{-4}$, which is more than adequate for present purposes.

The accuracy of the method is checked by performing some calculations with even smaller step-sizes and checking that the same results are obtained. It is found that for the initial conditions chosen, the system of differential equations displays no problematic 


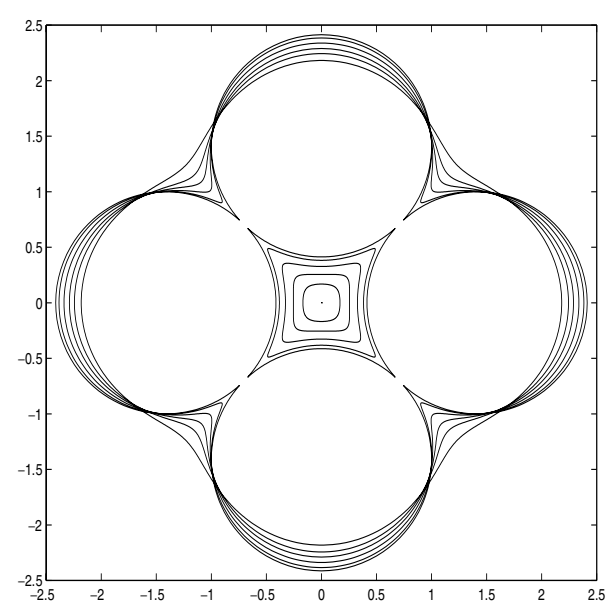

Figure 2. Case $N=4$ for times $t=0.2,0.4,0.6,0.8,1.143$.

stiffness problems at any point in the calculations even though this might be expected at early times when the fluid domain possesses several points of high curvature. An important check on the numerical method is provided by the following observation. Consider the map (2.16) with the parameter choices $\rho(0)=0.5, a(0)=1.5, A(0)=1$ and $N=50$. By plotting the image under this conformal map it is found to be indistinguishable (at least to the eye) from a concentric annulus $0.5<|z|<1.0$ even though there are small wrinkles on each near-circular boundary. Because the mathematical problem is well-posed, we expect these small wrinkles to smooth out quickly (Kuiken [15] has studied the damping of small ripples of this general kind on a flat interface and has shown that they die out exponentially quickly), and the disappearance time for the hole to be well approximated by the time taken for the disappearance of the hole in the simple concentric annulus solution which can be written down exactly. Indeed, this very check was performed by Van de Vorst [28] to test the accuracy of his boundary element method. The theoretical pore disappearance time based on the exact solution is $\sqrt{3}-1=0.73205 \ldots$ (see Van de Vorst [28]). The value obtained by the numerical procedure described above is 0.732 (correct to three decimal places).

Examples of the evolution of the boundaries in the cases $N=4$ and $N=5$ are shown in Figures 2 and 3. This class of calculations is expected to provide important benchmarks to test future numerical codes or analytical results. We therefore record a table of our chosen initial conditions along with estimates of the times $t^{*}$ at which the pores eventually disappear. The relevant data are given in the table in Table 1 for $N$ between 1 and 10, while a graph to cases up to $N=50$ is shown in Figure 4. As just discussed, $t^{*}$ is the time by which the area of the pore has shrunk to the order of $10^{-8}$. Using a different approach and elliptic function conformal mappings, Richardson [22] has also calculated pore disappearance times for cases $N=3$ and 4 . Richardson reports $t=0.584$ for $N=3$ and $t=1.15$ for $N=4$. These disappearance times are in good agreement with those found here but are roughly $1 \%$ larger. This might be explained by the fact that Richardson claims to integrate his evolution equations up to the point when the pore 


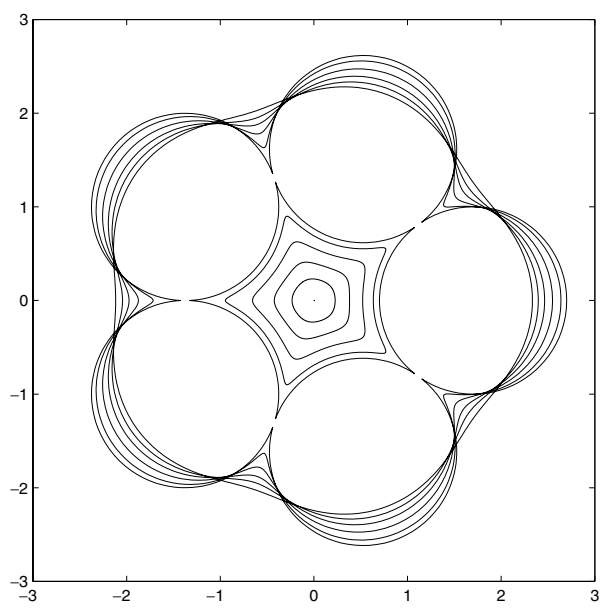

Figure 3. Case $N=5$ for times $t=0.3,0.6,0.9,1.2,1.637$.

Table 1. Table of initial conditions and pore disappearance times $t^{*}$

\begin{tabular}{rcccc}
\hline \hline$N$ & $\rho(0)$ & $a(0)$ & $A(0)$ & $t^{*}$ \\
\hline 3 & 0.3930 & 1.168 & 0.916 & 0.575 \\
4 & 0.5374 & 1.168 & 1.362 & 1.145 \\
5 & 0.6250 & 1.151 & 1.740 & 1.639 \\
6 & 0.6850 & 1.134 & 2.092 & 2.081 \\
7 & 0.7288 & 1.120 & 2.432 & 2.482 \\
8 & 0.7623 & 1.107 & 2.765 & 2.853 \\
9 & 0.7888 & 1.097 & 3.093 & 3.202 \\
10 & 0.8100 & 1.088 & 3.419 & 3.530 \\
\hline \hline
\end{tabular}

area is as small as $10^{-20}$, i.e. somewhat smaller than the final pore areas used here in calculating $t^{*}$.

A graph of the shrinking of the pore area as a function of time is shown in Figure 5. This graph is in qualitative agreement with similar graphs plotted on the basis of numerical boundary element calculations made by Van de Vorst [28]. A noteworthy feature of these results is that, for larger values of $N$, the shrinkage rate tails off quite dramatically as time evolves. This is because at the final stage of sintering the pore is observed to be close to circular with no points of high curvature so that pore shrinkage occurs more slowly.

Figure 6 shows a graph of neck radius defined as

$$
\frac{1}{2}\left|z\left(e^{\frac{i \pi}{N}}, t\right)-z\left(\rho(t) e^{\frac{i \pi}{N}}, t\right)\right|
$$

as a function of time for various values of $N$. It is interesting that this curve seems to be universal for all values of $N$. That is, the interparticle necks develop in exactly the 


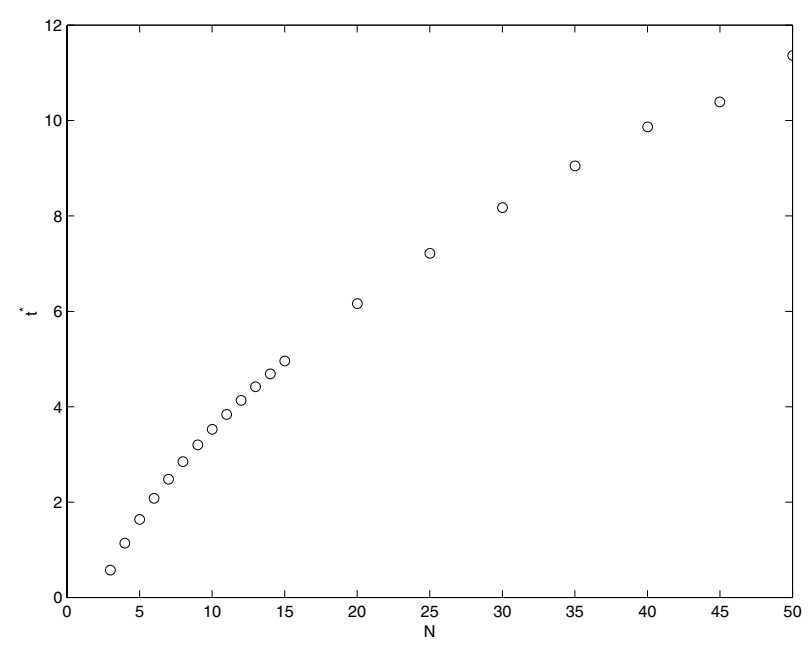

FIGURE 4. Disappearance times ( $y$-axis) against number of particles $N$ ( $x$-axis) (up to $N=50$ ).

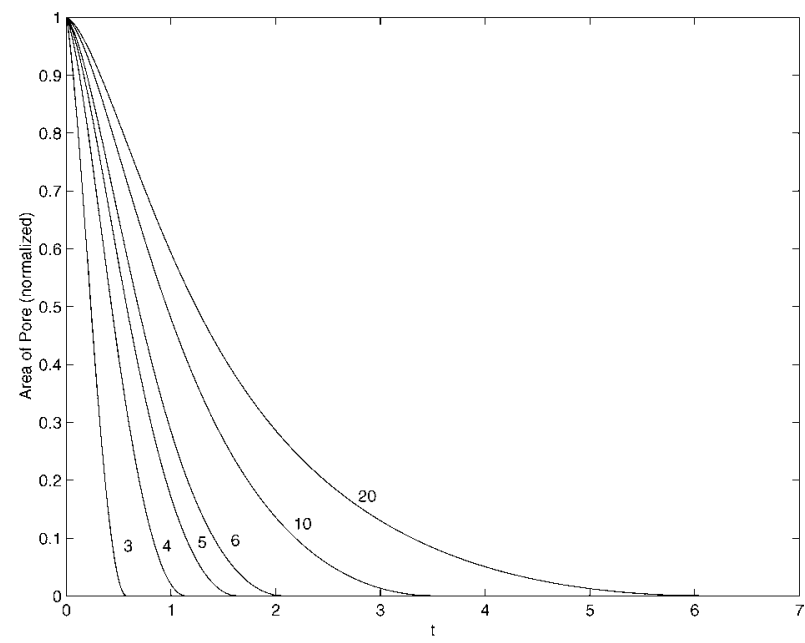

FIGURE 5. Shrinking of the enclosed pore for unimodal cylindrical packings with $N=3,4,5,6,10$ and 20. Areas are normalized with respect to initial pore area.

same fashion regardless of how many particles there are in the closed annular chain. A similar graph is plotted by Van de Vorst [28] for $N=3,4,5$ and 6 and he notes that the development of the neck radii is well described by Hopper's exact solution, thereby highlighting the usefulness of considering unit problems in viscous sintering. This universal behaviour also suggests that the early-stage neck growth might be well modelled locally by what has been dubbed a codimension-two free boundary problem [13, 10].

It is usual, in the sintering literature, to plot a quantity known as the relative density against reduced time. The shapes of such density-versus-time curves drawn on the basis 


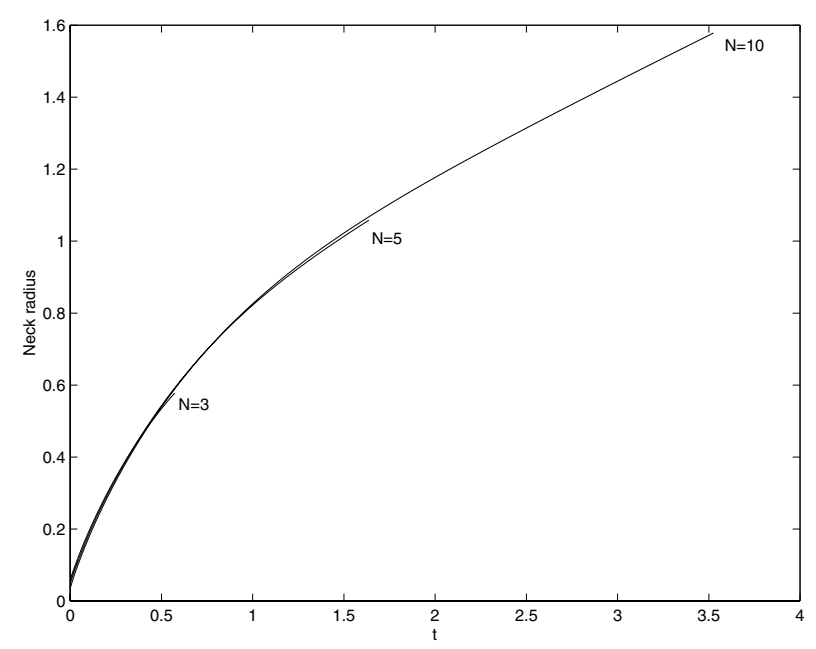

FIGURE 6. Evolution of neck radius for $N=3,4,5$ and 10 .

of experiments as well as the model systems described earlier are known to possess a distinctive sigmoidal shape (i.e. S-shaped). For typical graphs the reader is referred to Rahaman [18, Chapter 8]. It is of interest to examine whether the present calculations exhibit similar graphs. To do this, consider the special case $N=4$. It is then possible to identify a square 'unit cell' which is defined, at all times in the sinter process, by the four lines joining the points $z\left(a^{-1} \omega_{k}, t\right), k=0,1,2,3$ where $\omega_{k}=e^{\frac{i k \pi}{2}}$. See Figure 7 for a schematic. In a uniform square packing of equal-sized particles, this unit would be repeated in a regular lattice throughout the plane. As mentioned in the introduction, it will now be assumed that the global densification of the entire doubly-periodic compact is well described by considering the single isolated unit just defined (the extent to which this is true can only be tested by comparison with calculations of the full problem involving a doubly-infinite lattice - see also further comments in the conclusion section). Let $A_{f}(t)$ denote the area of fluid in the unit cell at time $t$. We then define the relative density $\sigma(t)$ at time $t$ as the ratio of the area of fluid inside this square unit to the total area of the square unit at time $t$, i.e.

$$
\begin{aligned}
\sigma(t) & =\frac{A_{f}(t)}{\text { Area of square unit }} \\
& =\frac{2 z\left(a^{-1}, t\right)^{2}-\frac{1}{2} \operatorname{Im}\left[\oint_{|\zeta|=\rho} \bar{z} z_{\zeta} d \zeta\right]}{2 z\left(a^{-1}, t\right)^{2}} .
\end{aligned}
$$

where $A_{f}(t)$ has been calculated by subtracting the numerically-computed pore area from $2 z\left(a^{-1}, t\right)^{2}$ which corresponds to the area of the square unit cell at time $t$. Note that the total area of the square unit varies in time because the compact as a whole is densifying. The reduced time $t_{r}$ is the time variable rescaled with the square root of the initial fluid 


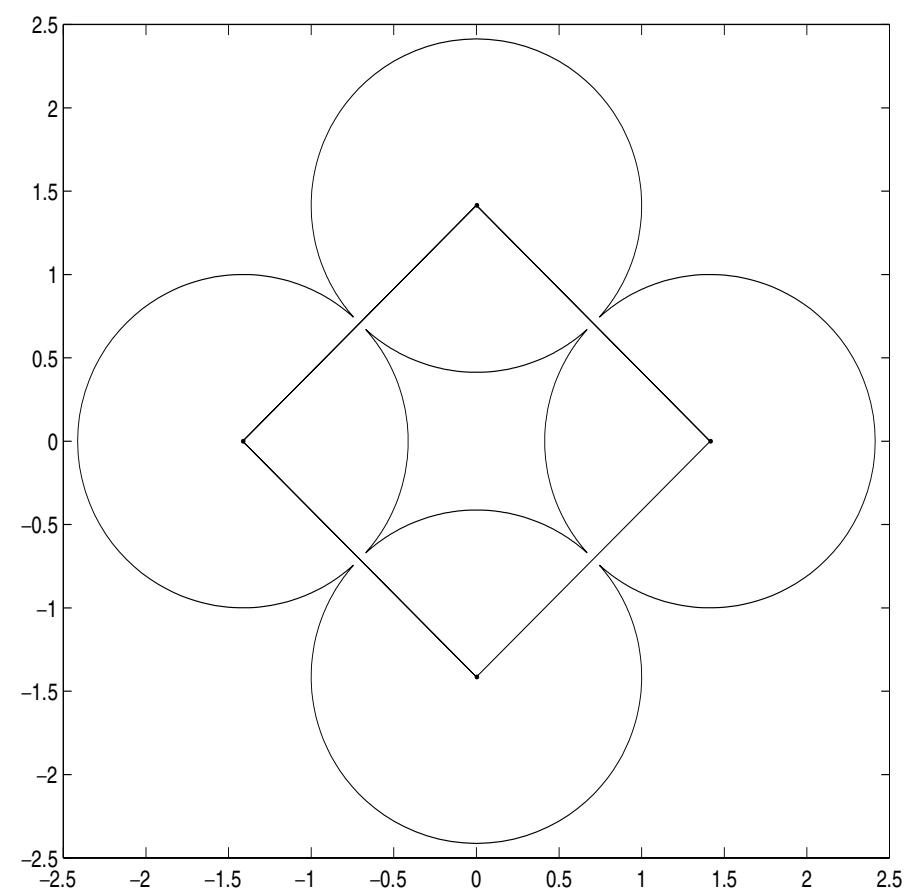

FIGURE 7. Schematic illustrating initial square unit cell for single particle size. Lines joining particle centres form the square unit cell.

area in the unit cell, i.e.

$$
t_{r}=\frac{t}{\sqrt{A_{f}(0)}} .
$$

Figure 8 shows a graph of the relative density $\sigma$ against reduced time $t_{r}$. This graph indeed displays the characteristic sigmoidal, S-shape typical of the density-versus-time data deduced from sinter experiments and models.

\section{Bimodal particle packings with pores}

As mentioned in the introduction, it is generally recognized that the viscous sintering of amorphous materials is not particularly sensitive to structural features of the sinter body such as the specific details of the initial pore shape. In this section, we use the exact solution class to examine whether this is also true in the model of planar sintering considered here.

We restrict attention to unit problems typified by a 'square unit' like the example considered at the end of $\S 3$. We now introduce a bimodal distribution of particles surrounding a single pore in an effort to study a range of square packings with distinct pore shapes and examine how these differing pore shapes affect the graphs of relative density against reduced time. The aim is to determine whether empirical estimates of the disappearance times of pores in arbitrary square planar packings can be obtained in a simple manner, without the need for detailed calculations in each separate case. 


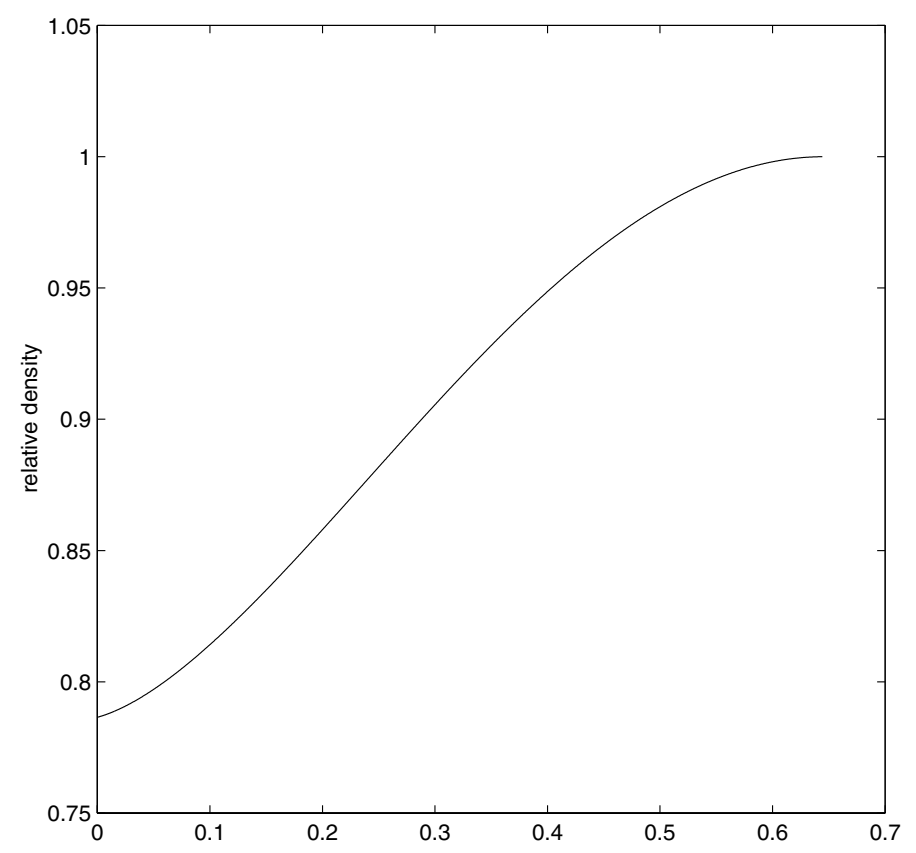

FIGURE 8. Graph of relative density against reduced time for $N=4$.

The conformal maps (2.17) provide the required alternating, bimodal distributions of cylindrical particles surrounding an enclosed pore. The initial configurations chosen are all close to the scenario depicted in Figure 9 in which four particles of smaller radius are placed between the particles in the $N=4$ case considered in the previous section in such a way that the centres of all the particles initially lie on the square joining four points $( \pm 1,0)$ and $(0, \pm 1)$. The relative radii of the two particle types are varied, the centre of the larger particles being placed at $( \pm 1,0)$ and $(0, \pm 1)$ and having radius $r$. Ten different cases are considered corresponding to choices of $r$ between 0.5 and 1 . Each initial configuration is chosen by finding the parameters $\rho(0), a(0), A(0), b(0)$ and $B(0)$ corresponding to the situation of eight touching circular discs (this is done using Newton's method to solve a system of nonlinear equations) and then perturbing these parameters in order to slightly smooth out the high curvature regions in the boundaries to obtain a connected configuration like that shown in Figure 9. This perturbation of the parameters in not done in a systematic way as in the previous section, but by simply tweaking the parameters corresponding to touching circular discs. The parameters used in the ten test cases (numbered 1-10) are listed in Table 2.

The class of conformal maps (2.17) depend on five real parameters. The five evolution equations are given by

$$
\begin{aligned}
& \dot{a}=a I\left(a^{-1}, t\right), \\
& \dot{b}=b I\left(\omega^{-1} b^{-1}, t\right),
\end{aligned}
$$




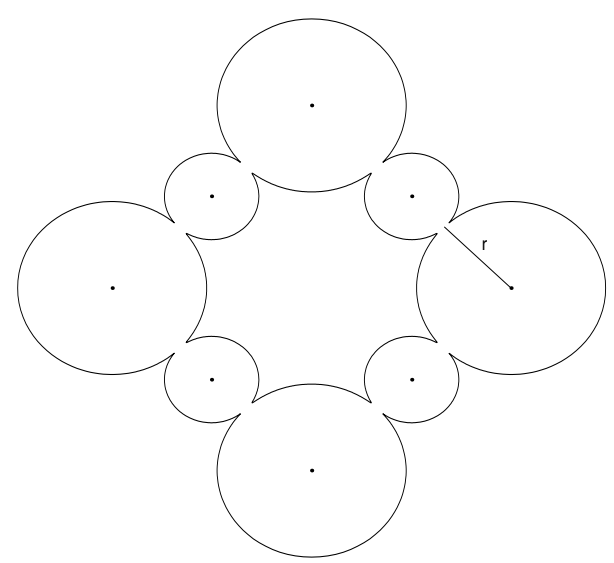

FIGURE 9. Schematic illustrating initial square unit cell with two particle sizes. Lines (not shown) joining particle centres (shown as dots) form the square unit cell.

Table 2. Table of initial conditions and reduced pore disappearance times for 10 example cases

\begin{tabular}{rccccccc}
\hline \hline Case & $\rho(0)$ & $a(0)$ & $A(0)$ & $b(0)$ & $B(0)$ & $r$ & $t^{*}$ \\
\hline 1 & 0.737 & 1.089 & 0.628 & 1.165 & 0.763 & 0.500 & 0.929 \\
2 & 0.669 & 1.099 & 0.661 & 1.210 & 0.677 & 0.567 & 0.886 \\
3 & 0.686 & 1.096 & 0.789 & 1.200 & 0.516 & 0.667 & 0.905 \\
4 & 0.709 & 1.088 & 0.828 & 1.184 & 0.450 & 0.707 & 0.901 \\
5 & 0.713 & 1.087 & 0.870 & 1.180 & 0.396 & 0.742 & 0.890 \\
6 & 0.696 & 1.090 & 0.900 & 1.184 & 0.343 & 0.778 & 0.854 \\
7 & 0.759 & 1.068 & 0.894 & 1.134 & 0.295 & 0.806 & 0.853 \\
8 & 0.700 & 1.092 & 1.005 & 1.187 & 0.231 & 0.849 & 0.810 \\
9 & 0.726 & 1.084 & 1.084 & 1.181 & 0.122 & 0.919 & 0.751 \\
10 & 0.811 & 1.054 & 1.064 & 1.117 & 0.066 & 0.955 & 0.718 \\
\hline \hline
\end{tabular}

where $\omega=e^{\frac{i \pi}{4}}$, the usual equation (2.10) for $\rho(t)$ and the two conserved quantities

$$
\begin{aligned}
\frac{A P_{N}\left(\rho^{2 / N}, \rho\right) z_{\zeta}\left(a^{-1}, t\right)}{N \hat{P}_{N}(1, \rho)} & =\text { constant }, \\
\frac{B Q_{N}\left(\rho^{2 / N} \omega^{-1}, \rho\right) z_{\zeta}\left(\omega b^{-1}, t\right)}{N \hat{Q}_{N}\left(\omega^{-1}, \rho\right)} & =\text { constant }
\end{aligned}
$$

where $\hat{Q}_{N}(\zeta, \rho)$ is defined as

$$
\hat{Q}_{N}(\zeta, \rho) \equiv \frac{Q_{N}(\zeta, \rho)}{\left(1+\zeta^{N}\right)}=\prod_{k=1}^{\infty}\left(1+\rho^{2 k N} \zeta^{N}\right)\left(1+\rho^{2 k N} \zeta^{-N}\right) .
$$




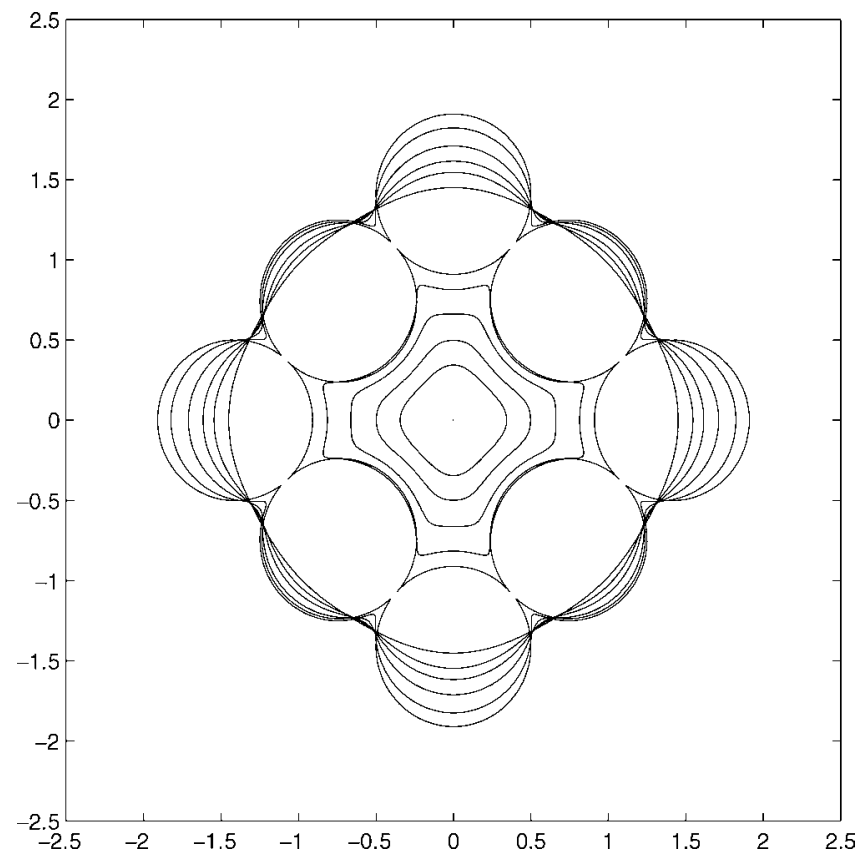

Figure 10. Case $1: t=0.0,0.2,0.4,0.6,0.8,1.367$

Plots of the initial conditions corresponding to cases 1,5 and 9 and their subsequent evolutions are shown in Figures 10-12. These figures show examples with increasing disparity between the radii of the two different particle sizes. The calculations were performed using a forward Euler method with time step 0.00025 and are terminated when $\rho$ is smaller than this step-size. The reduced shrinkage time $t^{*}$ for the ten test cases is reported in the table in Figure 2.

A graph of initial relative density against reduced shrinkage time of the pores is shown in Figure 13 as a scatter plot of the results for the ten cases given in the table in Table 2, as well as the $N=4$ case considered at the end of $\S 3$ (this case corresponds to $r=1$ ). It is clear that, except for a notable outlier corresponding to case 1, all points lie close to a straight line. This is significant; it suggests that the geometry of the interparticulate pores in a square packing is of little importance in actually calculating the total shrinkage time of the pores, which is the quantity of primary interest. Note also that all dots in Figure 13 correspond to different values of $r$ but, as can be seen from Figure 14 which contains a scatter plot of $r$ against initial relative density, different values of $r$ (and hence different initial pore shapes) can have similar initial relative densities and thus similar (reduced) shrinkage times. For example, cases 2 and 5 have very different initial shapes and yet their initial relative densities are roughly the same. They also have commensurate (reduced) pore disappearance times.

Under the hypothesis that the above results are presentative of the behaviour of pores in a general regular square packing of particles, the linear graph in Figure 13 can be used 


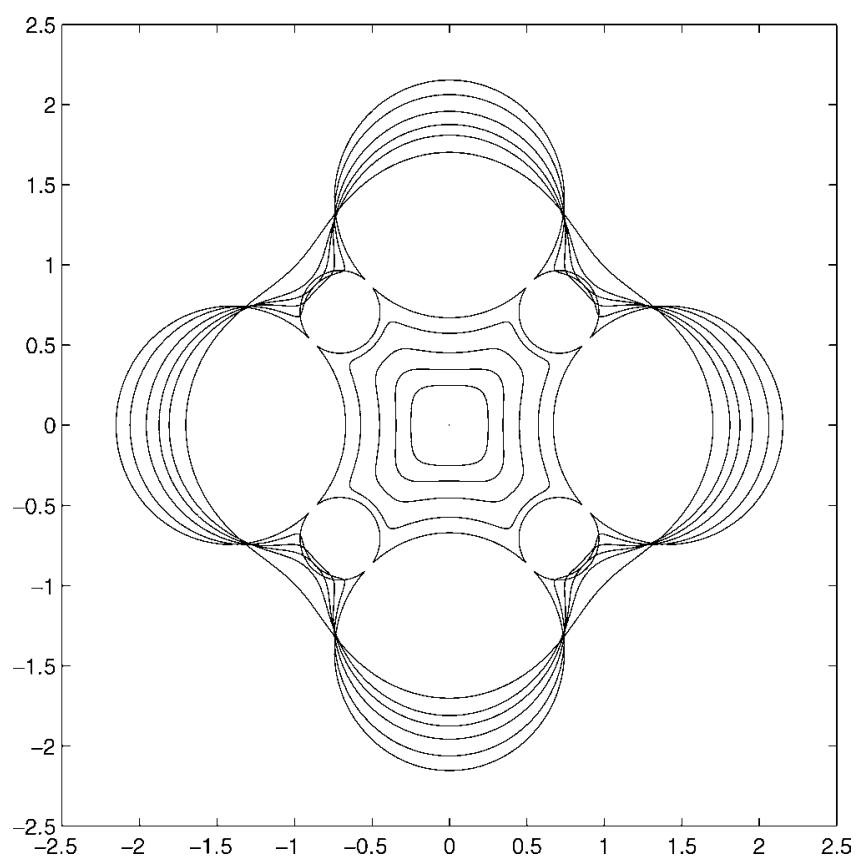

Figure 11. Case $5: t=0.0,0.2,0.4,0.6,0.8,1.302$.

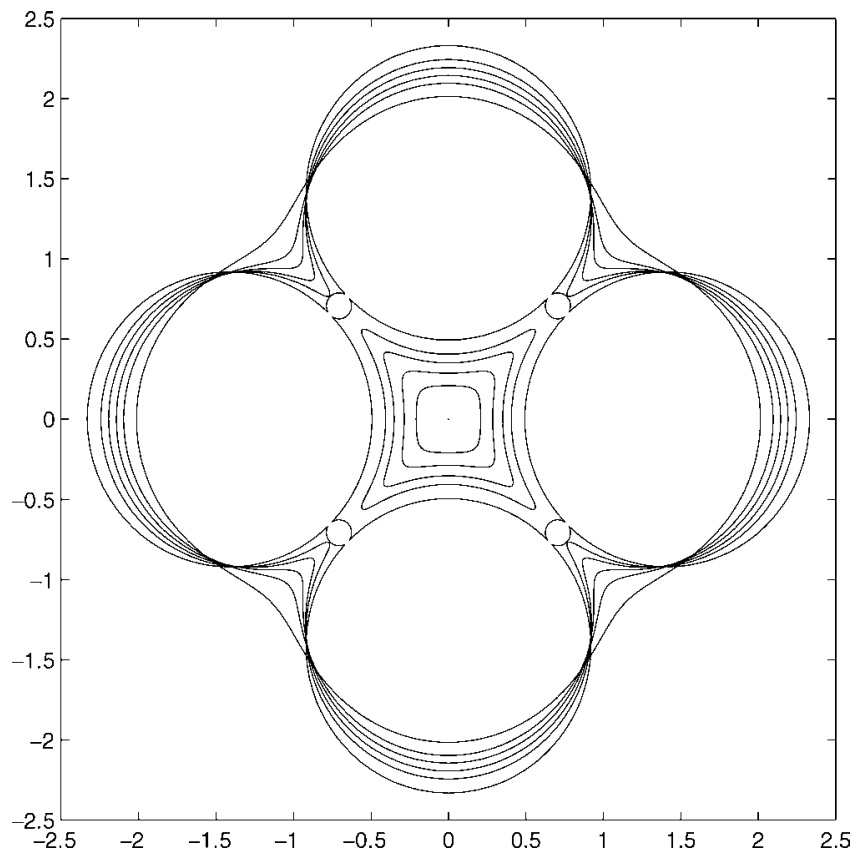

FIGURE 12. Case 9: $t=0.0,0.2,0.4,0.6,0.8,1.231$. 


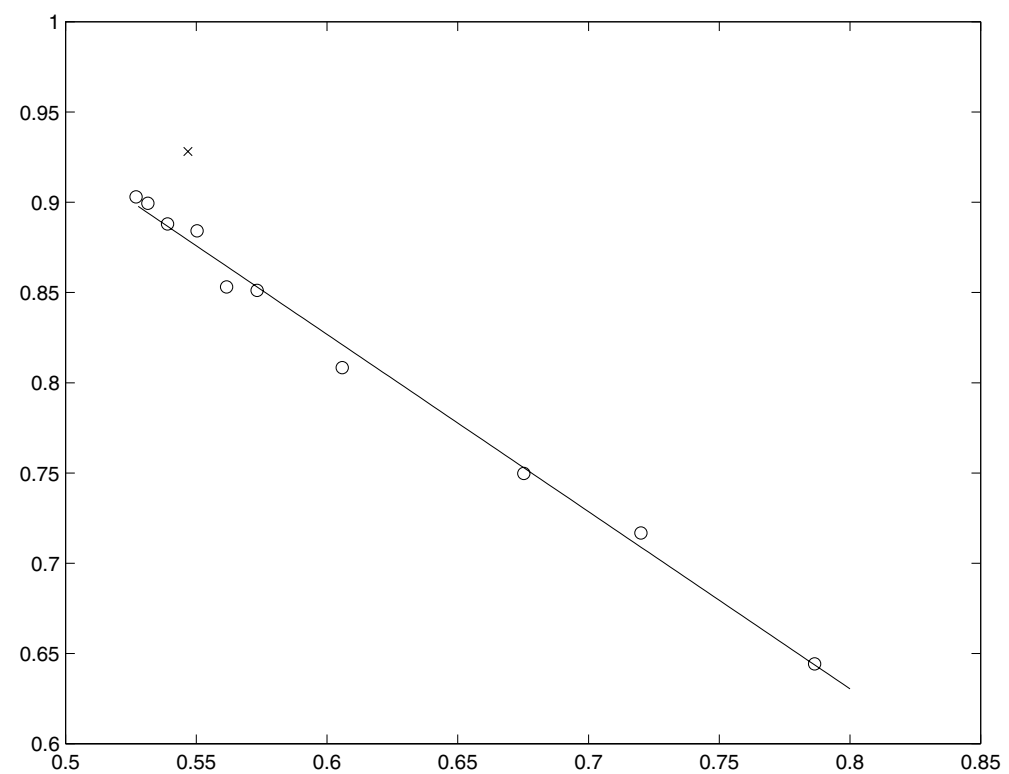

FiguRE 13. Graph of initial relative density ( $y$-axis) against reduced shrinkage times ( $x$-axis). Cross indicates outlier corresponding to case 1 , all other data shown as dots. Also shown is a best-fit line (4.4) through the dots.

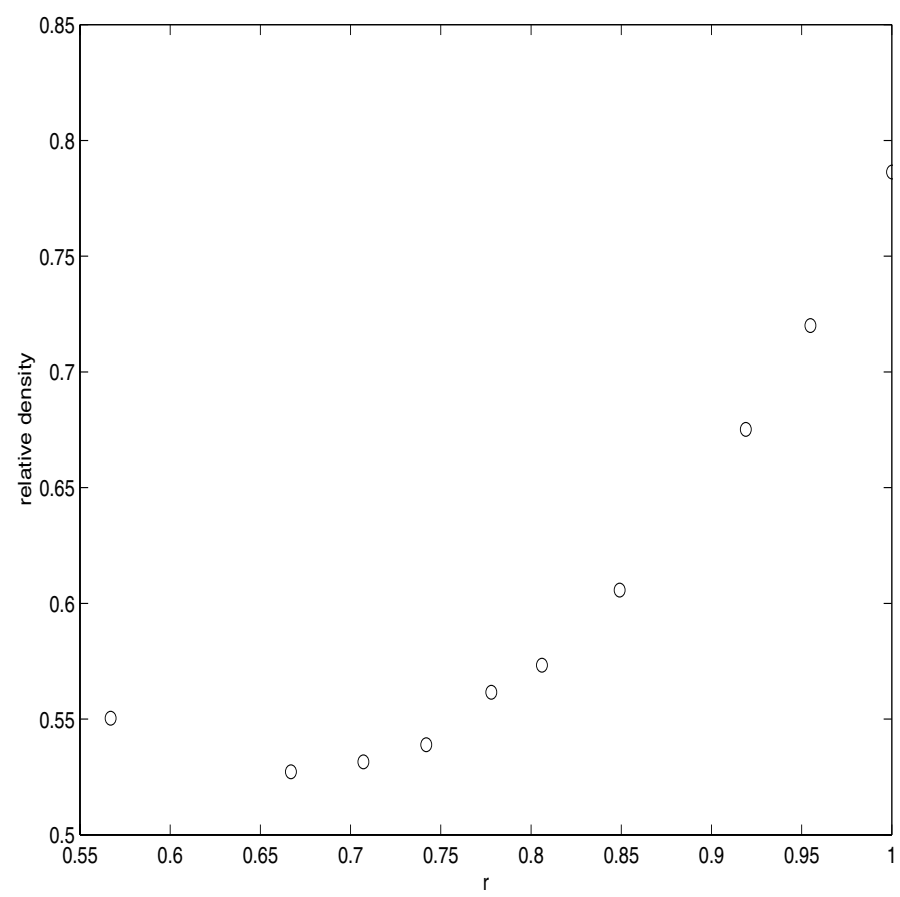

FIGURE 14. Scatter plot of $r$ against relative density. 


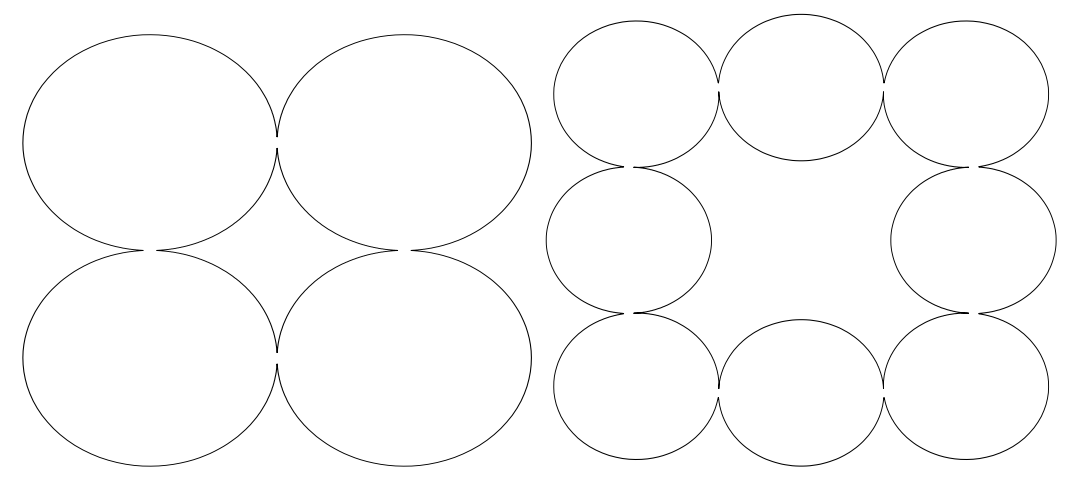

FIGURE 15. Two distinct square packings of equal-sized particles. If considered as a typical unit cell in a doubly-infinite lattice, the denser packing on the left has one particle per pore while the looser packing to the right has three particles per pore.

to provide quantitative predictions of disappearance time for an arbitrary square packing using nothing more than the initial size of a typical square unit cell and the initial relative density $\sigma(0)$. Disregarding the outlier corresponding to case 1 (this case will be studied in more detail shortly) a least-squares best fit line through the other ten data points in Figure 13 is found to be given by

$$
t_{f}=1.416-0.982 \sigma(0)
$$

This empirical relation, combined with estimates of the initial relative density (known in the engineering literature as green density $[1,18]$ ) and initial cell size (usually available experimentally) can be used to provide estimates of the time required for the sinter body to have densified so that pores are of negligible size.

Case 1 , corresponding to $r=0.5$, appears not to lie on the straight line (4.4) but takes a longer time for the pore to disappear than another configuration with the same initial relative density. Note that the choice $r=0.5$ results in another square unimodal packing which is distinct from the square unimodal packing considered in $\S 3$. If the square unit cell of touching circular particles is considered as a unit in a doubly-infinite lattice, the latter packing corresponds to a cell in which each pore is neighboured by a fluid region equivalent to one particle (i.e. the cell contains four quarter-particles) while the packing with $r=0.5$ has a pore neighboured by a fluid region equivalent to three particles (i.e. the unit cell contains four quarter-particles and four half-particles). The latter packing can therefore be described as being the looser packing because each pore is surrounded by more particles than the pore in the former packing. These two distinct square packings of equal-sized particles are shown in Figure 15. It is worth pointing out that 3-dimensional cubic packings of same-size particles which are analogues of the two distinct unimodal planar packings in Figure 15 are compared in Scherer $[1,26]$.

In an attempt to understand why case 1 does not follow the linear relation (4.4) particularly well, a graph of relative density against (shifted) reduced time for case 1 is 


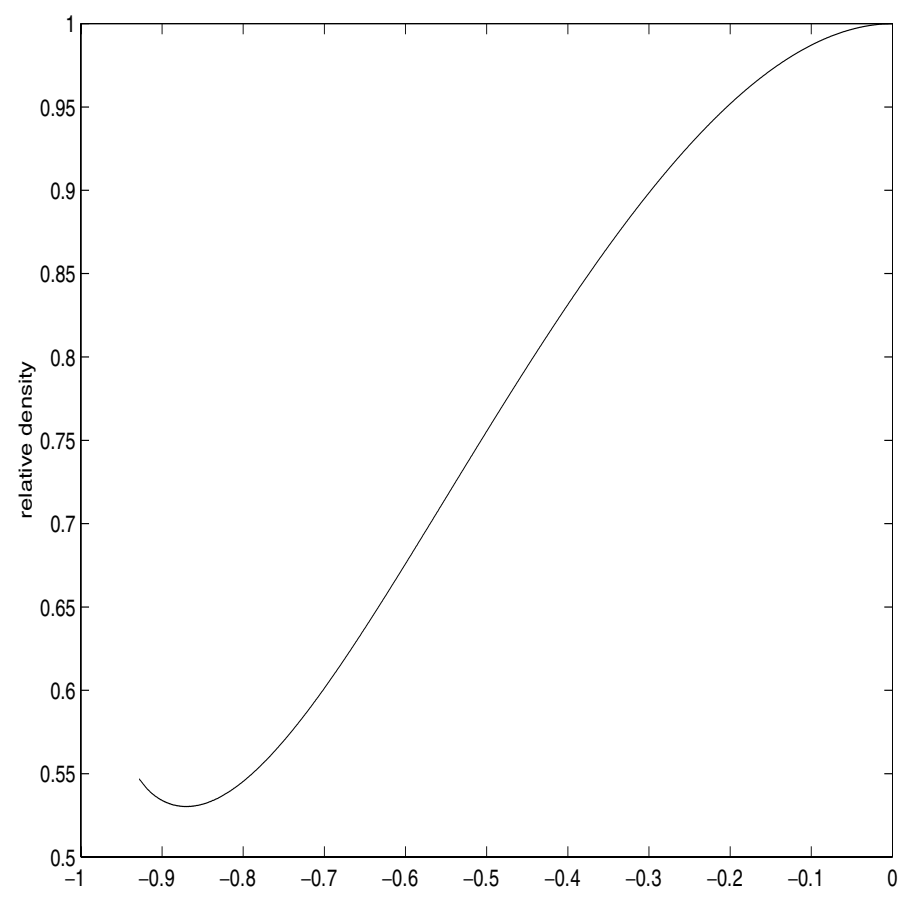

FIGURE 16. Relative density against shifted reduced time (abscissa) for case 1. Note the early period of dilation.

shown in Figure 16. Indeed, the abscissa corresponds to the quantity

$$
\frac{t-t_{f}}{\sqrt{A_{f}(0)}} .
$$

The most important feature of this graph is that, at early times, the relative density of the unit cell decreases. Indeed, the area of the enclosed pore is found to increase at early times before it eventually begins to decrease. If relative density is used to track densification under sintering, it must be concluded that this particular packing dilates, rather than densifies, in the early stage of sintering. This will have a negative effect on the densification rate. Van de Vorst [30] observed similar dilational behaviour in his numerical calculations of a texture model of a base-catalysed aerogel. He noticed that, in a doubly-infinite lattice of cells each containing a non-uniform distribution of pores, pores with large concave boundary parts become larger in size before shrinking and explains this phenomenon as a consequence of the fact that such pores have much longer boundary length than that strictly required to surround the given pore area. This argument would similarly explain the dilational behaviour of Case 1 observed here. It is clear from Figure 15 that the initial enclosed pore has many concave boundary parts.

It is also worth noting that Ross, Miller \& Weatherly [25] have performed computer simulations of two-dimensional sintering problems in order to study the effects of packing geometry on the sintering behaviour. Their results highlight the fact that in looser packings, overall densification can be inhibited even though interparticle shrinkage (i.e. 
growth of the necking regions between touching particles) still takes place. They explain this strong dependence of shrinkage on packing density by means of a 'chain straightening' mechanism. The calculations of Ross et al. [25] is based on a simulated model; the above calculations show that similar phenomena are present in exact integrations of the Stokes flow model with surface tension even for the simple unit problems considered here.

It is of interest to examine whether after this initial short period of dilation, the configuration follows the empirical relation (4.4). The following parameter choices describe the case 1 configuration at some time just after the dilation period:

$$
\rho=0.624 ; a=1.138 ; A=0.661 ; b=1.229 ; B=0.791 .
$$

The initial relative density of this configuration is $\sigma(0)=0.530$. Relation (4.4) then predicts a shrinkage time of 0.897 . Integration of the exact equations of motion from the initial conditions (4.6) gives the value 0.906. The empirical relation (4.4) therefore gives an excellent approximation to the disappearance time once the initial period of dilation has taken place.

\section{Conclusion}

Using a class of exact solutions, the results of this paper suggest that, provided one avoids pores with too many concave boundary parts, pore shrinkage times in the two-dimensional viscous sintering of a square packing are largely independent of the particular details of the initial pore shape and can be predicted to good accuracy by an empirical relation given the initial green density and cell size.

With recent advances in the use of boundary integral methods, there has been a revival of interest in the numerical calculation of planar viscous sintering. Early computer simulations of dynamic sintering was performed using finite element methods by Ross et al. [24]. Kuiken [15] performed some important calculations using boundary element methods. These calculations were later improved upon by a number of other authors [27, 28]. Extensive numerical investigations have been performed by Van de Vorst [27][29] whose calculations include the evolution of two different square packings of 16 equal particles [28]. The two packings differed only in their initial green densities. Van de Vorst [28] points out that the only limitation on the application of the numerical method is computer resources, i.e. the calculations are computationally intensive. The importance of the present results is that they suggest that some simple empirical relation such as (4.4) might be used to obtain estimates for the different pore shrinkage times of the two different 16-particle packings given only their initial green densities. This could potentially obviate the need for detailed calculations in the practical matter of estimating pore shrinkage times for a given packing.

The problems considered here have been isolated unit problems. It has been a thesis of this paper that such unit problems may, in certain circumstances, be representative of the behaviour of a typical unit cell in an extended packing and, for this reason, the mathematical properties of these isolated units have been studied. The next important step is to establish to what extent this is true and to investigate, for example, how the general relation (4.4) is affected if the unit is immersed in an infinite lattice of identical units repeated throughout the plane. Of course, some differences are to be expected. Indeed, in 
numerical calculations of an array of non-uniform pores in both a finite two-dimensional fluid region [29] and in a doubly-infinite lattice [30], Van de Vorst has observed that pores vanish in order of their initial size in a doubly-infinite lattice while, in a finite domain, the larger pores shrink faster than smaller ones. Van de Vorst [30] attributes this difference to the additional stresses in the fluid caused by the outer boundary in the finite domain case. Moreover, Jagota \& Dawson [14] have remarked on a danger in the use of unit problems to model viscous sintering. They point out that making incorrect geometrical assumptions concerning a unit problem, or ignoring far-field effects, can lead to the conclusion that the sinter body dilates rather than densifies. In the exact planar solutions above, the evolution of the geometry has been solved self-consistently with the full flow field and still dilation is observed in the early stages of sintering, at least in the case of a loose particle packing. Whether this dilational behaviour would persist if the initial unit cell of test case 1 is repeated in a doubly-periodic lattice is an interesting open question. Recall that Van de Vorst [30] has already observed dilational behaviour of this kind in a doubly-periodic packing.

The model of the sintering problem considered here has a number of fortuitous mathematical properties $[11,19,2,3,5,6,22]$. For example, Crowdy has advocated the usefulness of considering the problem in the context of quadrature domain theory [5], which is closely related to an alternative approach based on the Cauchy transform of the fluid domains (see Crowdy [6]) - a perspective which has proved particularly useful in the study of other free boundary problems such as Hele-Shaw flows involving multiplyconnected regions (see, for example, Richardson [23] and Crowdy \& Kang [8]). Using such analytical perspectives, Crowdy [7] has recently identified exact solutions for the sintering of finite fluid domains with larger numbers of pores. These solutions depend on just a finite set of time-evolving parameters and provide a wide source of increasingly complex unit problems which can be studied in a straightforward way to gain insight into the behavioural features of the model.

There is a more general implication of our results. Historically, experiments and theoretical models have shown that three-dimensional viscous sintering is well-described by empirical laws in which the details of the exact pore geometry are not important. This analytical study based on exact mathematical solutions has revealed the same phenomenon in the case of a physically-simplistic planar sintering model. This suggests the potential usefulness of studying planar sintering models possessing the fortuitous mathematical properties mentioned above. It is plausible that general properties and observations made in the context of the planar problem might carry over to the full three-dimensional case which is much less tractable to study either analytically or numerically. In further support of this conjecture, Nie \& Tanveer [17] have studied the axisymmetric problem in the case of volumetric change and have found that it shares many general features of the planar problem. Thus while the planar problem may be less physical, it appears to represent a useful and tractable theoretical paradigm.

\section{Acknowledgements}

The author gratefully acknowledges support from National Science Foundation (grant numbers DMS-9803167 and DMS-9803358) and from the Nuffield Foundation. 


\section{References}

[1] Brinker, C. J. \& Scherer, G. W. (1990) Sol-Gel Science. Academic Press.

[2] Crowdy, D. G. \& Tanveer, S. (1998) A theory of exact solutions for plane viscous blobs. J. Nonlinear Sci. 8(3), 261-279.

[3] Crowdy, D. G. \& Tanveer, S. (1998) A theory of exact solutions for annular viscous blobs. J. Nonlinear Sci. 8(3), 375-400.

[4] Crowdy, D. G. \& Tanveer, S. (2001) Erratum to 'A theory of exact solutions for annular viscous blobs'. J. Nonlinear Sci. 11, 237.

[5] Crowdy, D. G. (1999) A note on viscous sintering and quadrature identities. Euro. J. Appl. Math. 10, 623-634.

[6] Crowdy, D. G. (2002) On a class of geometry-driven free boundary problems. SIAM J. Appl. Math. 62(3), 945-964.

[7] Crowdy, D. G. (2002) Exact solutions for the viscous sintering of multiply-connected fluid domains. J. Eng. Math. 42, 225-242.

[8] Crowdy, D. G. \& Kang, H. (2001) Squeeze flow of multiply-connected fluid domains in a Hele-Shaw cell. J. Nonlin. Sci. 11, 279-304.

[9] Frenkel, J. (1945) Viscous flow of crystalline bodies under the action of surface tension. J. Phys. (Moscow) 5, 385.

[10] Gillow, K. A. (1998) Codimension-two free boundary problems. DPhil thesis, University of Oxford.

[11] Hopper, R. W. (1985) Coalescence of two equal cylinders: exact results for creeping viscous flow driven by capillarity. J. Am. Ceram. Soc. 67, C262-264.

[12] Hopper, R. W. (1990) Plane Stokes flow driven by capillarity on a free surface. J. Fluid Mech. 213, 349-375.

[13] Howison, S. D., Morgan, J. D. \& Ockendon, J. R. (1997) A class of codimension-two free boundary problems. SIAM Rev., 39, 221-253.

[14] Jagota, A. \& Dawson, P. R. (1988) Micromechanical modeling of powder compacts - I. Unit problems for sintering and traction induced deformation. Acta. Metall. 36(9), 25512561.

[15] Kuiken, J. K. (1990) Viscous sintering: the surface-tension-driven flow of a liquid form under the influence of curvature gradients at its surface. J. Fluid Mech. 214, 503-515.

[16] Mackenzie, J. K. \& Shuttleworth, R. (1949) Phenomenological theory of sintering. Proc. Phys. Soc. Lond. 62, 833.

[17] Nie, Q. \& TANVEer, S. Private communication.

[18] Rahaman, M. N. (1995) Ceramic Processing and Sintering. Marcel Dekker.

[19] Richardson, S. (1992) Two dimensional slow viscous flows with time dependent free boundaries driven by surface tension. Euro. J. Appl. Math. 3, 193-207.

[20] Richardson, S. (1996) Hele-Shaw flows with time-dependent free boundaries involving a concentric annulus. Phil. Trans. Roy. Soc. Lond. A, 353, 2513.

[21] Richardson, S. (1997) Two dimensional slow viscous flows with time dependent free boundaries driven by surface tension. Euro. J. Appl. Math. 8, 311-329.

[22] Richardson, S. (2000) Plane Stokes flow with time-dependent free boundaries in which the fluid occupies a doubly-connected region. Euro. J. Appl. Math. 11, 249-269.

[23] Richardson, S. (2001) Hele-Shaw flows with time-dependent free boundaries involving a multiply-connected fluid region. Euro. J. Appl. Math. 12, 571-599.

[24] Ross, J. W., Miller, W. A. \& Weatherly, G. C. (1981) Dynamic computer simulation of viscous sintering flow kinetics. J. Appl. Phys. 52(6), 3884-3888.

[25] Ross, J. W., Miller, W. A. \& Weatherly, G. C. (1982) Computer simulations of sintering in powder compacts. Acta Metall., 30, 203-212.

[26] Scherer, G. W. (1977) Sintering of low-density glasses I: Theory. J. Am. Ceram. Soc. 60, 236.

[27] Van de Vorst, G. A. L., Mattheij, R. M. M. \& Kuiken, H. K. (1992) A boundary element solution for two dimensional viscous sintering. J. Comput. Phys., 100, 50-63. 
[28] VAN DE Vorst, G. A. L. (1993) Integral method for a two-dimensional Stokes flow with shrinking holes applied to viscous sintering. J. Fluid Mech. 257, 667-689.

[29] VAn DE Vorst, G. A. L. (1994) Modelling and numerical simulation of Viscous Sintering. PhD thesis, Eindhoven University of Technology.

[30] VAN DE VORST, G. A. L. (1996) Integral formulation to simulate the viscous sintering of a two-dimensional lattice of periodic unit cells. J. Eng. Math. 30, 97-118.

[31] Valiron, G. (1945) Cours d'Analyse Mathematique: Theorie des Fonctions, 2nd Ed. Masson et Cie, Paris. 
